\title{
A new approach to generate tailed-sperm and oocytes in vitro from the same mouse embryonic stem cells
}

\author{
Lixin Feng $^{1}$ \\ ${ }^{1}$ Shanghai Jiao Tong University School of Medicine, Shanghai, China
}

Embryonic stem (ES) cells have the pluripotency to differentiate into any cell types in vitro, including cells of the germ lineage. However, the mechanisms that lead ES cells to differentiate into germ cells in vitro remain unknown and the efficiency of reported in vitro systems is poor. We have attempted to find a more defined and efficient way to derivate sperm and oocytes from mouse ES cells in vitro by manipulating genes such as $\mathrm{Daz}$ and $\mathrm{Dazl}$. The gene Daz (deleted in azoospermia), which is essential for sperm development in humans, is expressed in ES cells, as well as in male and female germ cells. In mice, its homolog, $\mathrm{Dazl}$, is turned off once mouse ES cells undergo somatic differentiation, but its expression is maintained in germ cells. Dazl deficiency can lead to embryonic arrest of germ cell development in mice. Here, we report that Dazl as a transgene can promote gametogenesis of mouse ES cells. Overexpression of Dazl can induce both tailed-sperm and oocytes from the same mouse ES cells in vitro. This finding may provide a novel in vitro approach to generate gametes from embryonic stem cells and to study gametogenesis of embryonic stem cells.

Keywords: embryonic stem cells; germ cells; sperm; oocytes; dazl

Cell Research (2008) 18:s17. doi: 10.1038/cr.2008.107; published online 4 August 2008

Correspondence: Lixin Feng

E-mail: fenglx66@yahoo.com

Lixin Feng, PhD in biochemistry ( from Peking Union Medical College in 1996), Pricinpal Investigator, Institute for Medicine Research, Shanghai Jiao Tong University School of Medicine. Dr Feng's research interests concern with the molecular mechanisms regulating the fate of spermatogonial stem cells (SSCs) and germline differentiation of embryonic stem cells. He revealed the signaling pathway that regulate the proliferation of SSCs as well as a new approach to derive both sperm and oocytes from embryonic stem cells in vitro. 